\title{
L'utilité des endoprothèses encore plus élevée que leur coût
}

\author{
Josef E. Brandenberg
}

Dr méd., spécialiste en orthopédie et traumatologie, membre $\mathrm{FMH}$, président fmCh

Les interventions chirurgicales pour prothèses du genou ou de la hanche ont souvent mauvaise presse, notamment en raison des coûts engendrés par l'accroissement de ces opérations. Or des patients de tous âges profitent incontestablement de ces interventions qui leur permettent une vie active et sans douleurs, en leur évitant la dépendance, ce qui finalement se traduit aussi par des économies de coûts.

La médecine chirurgicale et invasive suscite souvent une réaction ambiguë dans l'opinion publique, étant à la fois "efficace et dangereuse», relève la fmCh, l'association suisse des médecins avec activité chirurgicale. L'écho médiatique de leur impact positif reste faible, alors que les risques et les fautes professionnelles font couler beaucoup d'encre. Plutôt que de parler bénéfices, les médias et les politiciens insistent principalement sur les coûts: les maladies de l'appareil locomoteur sont responsables de $15 \%$ des séjours hospitaliers et représentent la principale cause d'hospitalisation [1]. Et comme les implantations de prothèses du genou et de la hanche ont fortement augmenté ces dernières années [1], la cause de la hausse des dépenses de santé est souvent imputée à des opérations qui auraient été précipitées.

\section{Le bénéfice individuel de vieillir en bonne santé offre également un intérêt collectif}

L'observation plus attentive des patientes et des patients traités révèle de nombreuses autres motivations pour cette augmentation des traitements, ainsi que d'importants bénéfices. La catégorie de patients concernée par les endoprothèses n'est pas la seule à augmenter avec le vieillissement de la population: les personnes âgées étant de plus en plus actives, les problèmes de genou ou de hanche représentent des limitations de mouvements plus marquantes et entraînent une plus forte demande pour ce type d'opérations. Comme parallèlement les risques opératoires diminuent avec le progrès médical, de plus en plus de patients y ont accès, alors qu'ils n'auraient pas pu être opérés il y a encore quelques années. Le maintien d'une vie active et sans antidouleurs améliore considérablement la qualité de vie des personnes concernées et a un impact positif sur leur santé physique et psychique.

Sur la liste OMS de «l'espérance de vie en bonne santé» [2], la Suisse figurait en 2007 avec 73,3 ans à la troisième place derrière le Japon et la Suède, avant nos voisins, l'Italie, la France, l'Autriche et l'Allemagne, mais aussi avant les Pays-Bas ou encore l'Angleterre et son National Health Service qui pourtant nous sont toujours cités en exemple; les Etats-Unis, avec une espérance de vie moyenne de 69,3 ans, sont nettement distancés. En 2012, l'espérance de vie en bonne santé en Suisse était de 77,5 ans pour les hommes et de 77,9 ans pour les femmes [3].

L’impact sur la société de ce bénéfice individuel de vieillir en bonne santé reste en Suisse largement sousestimé. Une étude américaine montre qu'avec la diminution des entrées en EMS et des médicaments, la prothèse de la hanche implantée engendre un gain annuel de 50000 USD [4]. Et cela sans compter la réduction des incapacités de travail.

\section{Une nouvelle articulation du genou ou de la hanche permet des économies importantes en soins et frais d'invalidité}

Comme la recherche sur les soins faite en Suisse contrairement à l'étranger [5-8] - reste un parent pauvre, nous ne disposons malheureusement pas de données nationales sur les bénéfices réalisés grâce aux endoprothèses. Mais analysons la relation coûts-utilité sur la base de deux exemples:

Madame W. vivait avec sa fille de 80 ans dans une maison d'une commune rurale: le logement était réparti sur deux étages, avec un escalier étroit, sans ascenseur. Malgré ses 102 ans, elle s'occupait du jardin, cuisinait et 
partait se promener, jusqu'au jour où de vives douleurs au genou l'ont immobilisée chez elle. La radiographie a alors révélé une ostéonécrose du condyle fémoral, soit la mort du tissu osseux du fémur (os de la cuisse). Fautil encore opérer à cet âge? Le médecin de famille, l'anesthésiste et le cardiologue y étaient favorables. On a donc mis en place une prothèse du genou. Au bout de six semaines, Madame W. était rentrée chez elle, et a pu y vivre encore 5 ans, avant d'être emportée par une pneumonie.

Le séjour hospitalier, l'opération, l'implantation, l'anesthésie, la consultation chez le cardiologue ainsi que les quatre semaines de réadaptation ont coûté au total 60000 francs. Un placement dans un EMS public de la commune aurait coûté environ 300 francs par jour, estimés pour un niveau de soins moyens (BESA 6). La caisse-maladie et les pouvoirs publics auraient contribué à ces coûts à hauteur de 190 francs. Pour une durée de séjour allant jusqu'au décès, cette solution aurait coûté 200000 francs à Madame W., et 347000 francs à la caisse-maladie et à la commune. Un cynique pourrait se demander si elle ne serait pas décédée plus tôt sans opération. C'est bien évidemment possible, mais on ne meurt pas si vite d'une ostéonécrose. Au bout de six mois, la vie de qualité moyenne en EMS avec incapacité à marcher et analgésiques aurait déjà coûté plus cher que la variante opératoire.

Le second cas est celui d'une factrice de 25 ans, chez laquelle une dysplasie bilatérale de la hanche - soit une malformation de l'articulation de la hanche - n'avait pas été diagnostiquée à la naissance. Une arthrose avancée des deux hanches risquait aujourd'hui d'entraîner une incapacité de travail. Malgré son jeune âge, on décida de lui implanter une prothèse de la hanche de chaque côté, ce qui a engendré des coûts de 80000 francs. La jeune femme a pu à nouveau marcher sans douleurs et sa qualité de vie s'est indiscutablement améliorée. Et quelle utilité pour la société? Depuis 15 ans, la patiente peut continuer à exercer son métier. Il suffit de calculer ce qu'auraient coûté une réorientation et réinsertion professionnelles, ou le versement d'une rente! Entre ces deux cas d'âges extrêmes, il y a chaque année 17000 personnes qui sont opérées pour une prothèse de hanche et 13000 pour une prothèse de genou dans les hôpitaux suisses [9]. Toutes ces personnes peuvent ensuite mener une vie autonome et sans douleurs, vaquer à leurs occupations et exercer leur métier, et participer au développement économique et social en tant que sportif, touriste ou consommateur. S'il nous faut évaluer les soins aux patients sous l'angle économique, nous devons aussi mettre en balance les bénéfices pour l'individu et la société par rapport aux coûts des traitements.

\section{Résumé}

Les coûts induits par la hausse des prothèses du genou et de la hanche font l'objet de nombreuses critiques. Si l'on considère cependant le nombre croissant de patients qui bénéficient de ces interventions, on constate que ces opérations présentent également une bien plus large utilité. Si la reprise d'une vie active sans analgésiques améliore la santé physique et psychique générale des personnes traitées, elle présente également un intérêt économique considérable en évitant ou repoussant chez ces personnes l'incapacité de travailler et la dépendance aux soins. Même chez une patiente de 102 ans, des coûts de traitement de 60000 francs «en valent la peine», y compris du point de vue économique, dès lors que six mois de soins en institution sont économisés. Des prothèses onéreuses sont encore plus «rentables» lorsqu'elles permettent à de jeunes personnes de préserver leur aptitude au travail. Ces bénéfices méritent une plus grande attention dans le débat sur les coûts de la santé.

\section{Références}

1 Office fédéral de la statistique (OFS). Statistiques de la santé 2014 Office fédéral de la statistique (OFS) Neuchâtel, 2014.

2 OMS, Statistiques sanitaires mondiales 2007.

3 Interpharma (2017). Système suisse de santé. 8-9.

4 George L, Ruiz D, Sloan F (2008). The effects of total hip arthroplasty on physical functioning in the older population. J Am Geriatr Soc. Jun;56(6):1057-62.

5 Räsänen P, Paavolainen P, Sintonen $\mathrm{H}$, Koivisto AM, Blom M, Ryynänen OP, et al. (2007). Effectiveness of hip or knee replace ment surgery in terms of quality-adjusted life years and costs. Acta Orthop. Feb;78(1):108-15.

6 George LK, Hu L, Sloan FA (2014). The effects of total knee arthroplasty on physical functioning and health among the under age 65 population. Value Health. 2014 Jul;17(5):605-10.

7 Sloan FA, George LK, Hu L (2014) Productivity Improvements in Hip and Knee Surgery. Arthritis. 2014615784.

8 Koenig L, Zhang Q, Austin MS, Demiralp B, Fehring TK, Feng C, et al. (2016). Estimating the Societal Benefits of THA After Accounting for Work Status and Productivity: A Markov Model Approach. Clin Orthop Relat Res 474.2645-54.

9 SIRIS, Swiss National Joint Registry, Report 2012-2015:25-6. 